\title{
Métaux et Médecine du Travail
}

\section{Metals and occupational medicine}

\section{Betty DEHON*(1), Catherine NISSE ${ }^{(2)}$, Michel LHERMITTE ${ }^{(1)}$, Jean-Marie HAGUENOER ${ }^{(2)}$}

(1) Laboratoire de Biochimie et Biologie Moléculaire, Hôpital Calmette, CHRU - 59037 LILLE Cedex 2

(2) Laboratoire de Médecine du Travail, CHRU - 59045 LILLE Cedex

* Auteur à qui adresser la correspondance : Betty DEHON, Laboratoire de Biochimie et Biologie Moléculaire,

Hôpital Calmette, CHRU de Lille - 59037 LILLE Cedex

Tél : 0320444969 - Fax : 0320444927 - E-mail : b-dehon@chru-Lille.fr

\section{RÉSUMÉ}

Le but de ce chapitre est de rapporter les risques encourus lors d'une exposition professionnelle à différents métaux. Dans un programme de prévention, trois types de surveillance doivent être mis en ceuvre : la surveillance de l'environnement avec les valeurs limites d'exposition (VLE et VME), la surveillance biologique de l'exposition, élément déterminant dans la protection de l'homme dans son activité professionnelle contre les effets adverses des métaux et le dépistage précoce des effets toxiques, condition préalable à l'évaluation du risque pour la santé.

\section{SUMMARY}

The aim of this paper is to evaluate risks encountered during professional exposure to different metals. In a preventive process, three types of surveillance are defined: the environmental management with the determination of threshold limit values (VLE and VME), the biological surveillance of the exposure which is a determinant element in the human protection from the adverse effects of metal during a professional activity and the early detection of toxic effects which is a previous condition for the estimation of the human health risk. 


\section{Introduction}

Parmi les missions de la Médecine du Travail, celles qui sont en relation avec les risques toxicologiques concernent : la surveillance de la santé des travailleurs et, tout particulièrement le dépistage des effets nocifs liés au travail (prévention des accidents du travail, des maladies à caractère professionnel), et l'étude des conditions de travail, des risques et des nuisances.

Parmi les nombreuses substances auxquelles sont exposés les salariés, les métaux et leurs dérivés occupent une place importante.

La première partie de cette revue présente pour chaque métal : les sources potentielles d'exposition, les manifestations cliniques, la surveillance biologique qui constitue une démarche complémentaire de la surveillance atmosphérique pour apprécier l'exposition des travailleurs aux différentes substances.

La seconde partie rapporte les effets des principaux métaux et les méthodes de surveillance mises en œuvre dans la surveillance des salariés.

\section{Présentation Générale Sources d'exposition potentielle}

Les principales sources d'exposition sont industrielles, domestiques et automobiles

- Industries minières, métallurgie des métaux non ferreux

- Bâtiment (soudeurs, serruriers), réhabilitation d'immeubles vétustes (peintures au plomb)

- Transformation des métaux

- production d'alliages (cuivre, sélénium, manganèse, nickel, ...)

. brasage : étain, argent, cadmium

. soudure : aluminium, cuivre, nickel, plomb, zinc.

- Production de batteries (plomb, ..), réparation de radiateurs

- Peinture, pigments pour peintures, céramique (cadmium, plomb, aluminium, cuivre, ...)

- Stabilisants dans l'industrie des matières plastiques (cadmium, plomb)

- Tannerie (chrome, arsenic, ...)

- Extraction de l'or (mercure)

- Sols contaminés (plomb, cadmium, cuivre, zinc, arsenic, ...)

- Fumée de tabac (cadmium).

\section{Métabolisme des métaux}

Selon la nature physico-chimique ou les conditions d'utilisation du métal ou de ses dérivés, l'absorption peut être :

- pulmonaire (fonction de la taille des particules et fonction de la spéciation),

- digestive : selon la taille des particules (granulométrie).

En effet, certains métaux (plomb, cadmium) rejetés par des centrales thermiques ou métallurgiques sont transportés par le vent sous forme d'aérosols. Par la suite, la pluie dépose ces contaminants sur le sol ou dans les cours d'eau, où certains d'entre eux s'accumulent et se transforment. La figure 1 illustre le devenir de certains métaux dans l'environnement.

Le cadmium présent dans les engrais ou dans les boues d'épuration est susceptible d'être assimilé par les plantes cultivées. De même, des déchets minéraux tels les chromates peuvent détruire la faune et la flore à de faibles doses. Certains métaux bioaccumulables (mercure, cadmium, plomb) peuvent atteindre l'homme à travers la chaîne alimentaire depuis le plancton.

Après s'être déposé sur le sol, le «métal» peut être retenu par les matières organiques, il y aura solubilisation et transfert. C'est souvent par les végétaux (feuille, herbe, fleur, lichens) que commence la chaîne alimentaire. Les herbivores (mammifères, insectes ou escargots) se nourrissent de végétaux en les mangeant, en les butinant ou en buvant la sève. Les carnivores ou insectivores se nourrissent des herbivores.

Après ingestion, la biodisponibilité sera différente selon la spéciation du métal (exemple le sulfure de plomb, oxyde de plomb). Les taux d'absorption sont différents aussi suivant l'âge, les enfants absorbent plus de plomb $(50 \%)$ que l'adulte $(10 \%)$.

Le métal est ensuite transporté dans le sang et stocké au niveau de l'organe cible, variable selon le métal, cadmium : rein ; arsenic : peau ; chrome : peau ; plomb : os.

\section{Les manifestations cliniques (1)}

Elles sont propres à chaque métal et sont résumées dans le tableau I. Elles sont reprises pour certains des métaux dans le paragraphe spécifique à celui-ci.

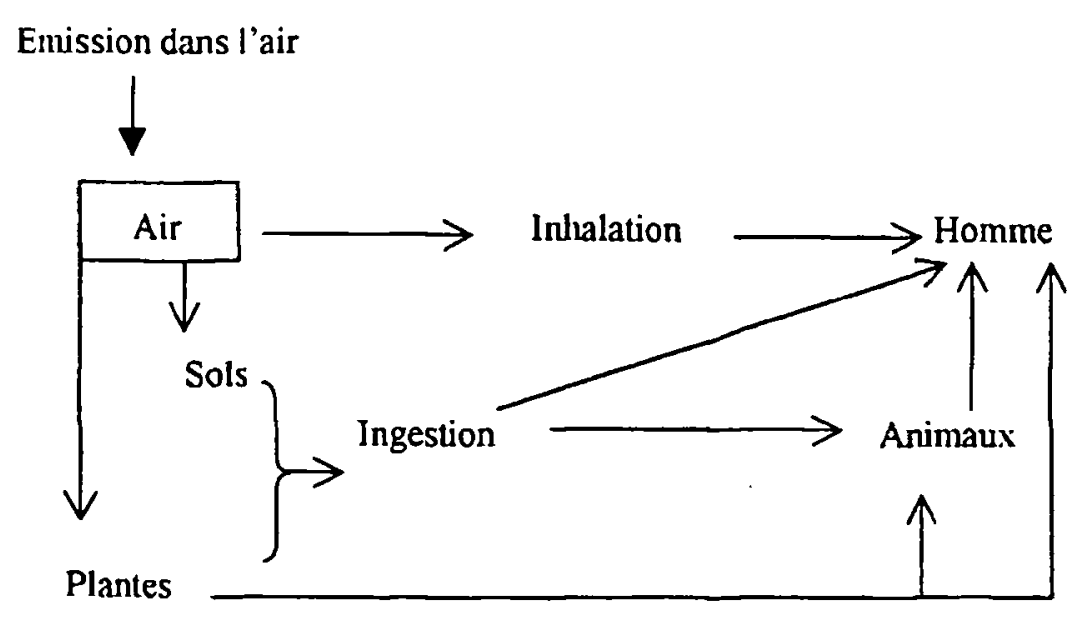

Figure 1 : Devenir des métaux dans l'environnement. 
Tableau I : Principales manifestations cliniques.

\begin{tabular}{|l|l|}
\hline Arsenic & $\begin{array}{l}\text { "Choléra arsenical", mélanodermie, polynévrite, cirrhose, toxicité cardiaque, } \\
\text { atteinte des ongles, cheveux et muqueuses, cancer de la peau, des poumons }\end{array}$ \\
\hline Beryllium & Conjonctivites, dermites, pneumopathie, fibrose pulmonaire \\
\hline Cadmium & $\begin{array}{l}\text { Fièvre des fondeurs, asthme, atteinte rénale, troubles gastro-intestinaux, } \\
\text { néoplasme des bronches et des fosses nasales. } \\
\text { Déminéralisation osseuse }\end{array}$ \\
\hline Chrome & $\begin{array}{l}\text { Ulcération de la cloison nasale, pigeonneaux des doigts, dermite de contact, asthme, } \\
\text { Bronchopneumopathies obstructives chroniques (BCPO), emphysème } \\
\text { néoplasme des bronches et des fosses nasales }\end{array}$ \\
\hline Cobalt & Fibrose pulmonaire, allergie \\
\hline Nickel & Fièvre des fondeurs, pneumopathie, dermite eczématiforme \\
\hline Plomb & Saturnisme, encéphalopathie, lésions rénales, anémie \\
\hline
\end{tabular}

\section{Surveillance biologique}

L'exposition aux substances chimiques de l'environnement professionnel est un sujet en plein développement en raison de la modification régulière des indices biologiques d'exposition internationaux et de l'apparition récente de valeurs guides utilisables en France.

Ces dosages biologiques apportent également leur contribution à l'évaluation des risques environnementaux pour l'homme par la mise en place des programmes de surveillance biologique des populations exposées.

La surveillance biologique d'exposition consiste à mesurer dans les milieux biologiques (sang, urine) de travailleurs exposés à des métaux des indicateurs biologiques d'exposition IBE (le toxique lui-même, ou produits de transformation ou métabolites). De plus, les indices biologiques d'exposition (BEI) proposés par les hygiénistes américains de l'American Conference of Gouvernmental Industrial Hygienists (ACGIH) et allemands (BAT) de Deutsche ForschungsGenanschaft (DFG) sont des moyens d'apprécier les expositions des travailleurs

En France, les indicateurs biologiques n'ont pas de caractère réglementaire, sauf pour le plomb.

La surveillance de l'exposition peut donc être abordée de plusieurs façons : d'une façon globale par la surveillance des atmosphères de travail (mesure des concentrations du produit dans l'environnement de travail comparée à des normes de référence ; VLE : valeur limite d'exposition pour des durées inférieures ou égales à $15 \mathrm{~min}$. Leur respect prévient les risques d'effets toxiques immédiats ou à court terme ; VME : valeur moyenne d'exposition pour une période de référence de 8 h, 5 jours par semaine. Elle est destinée à protéger les travailleurs des effets à long terme) et d'une façon individuelle par la mesure chez l'individu lui-même de la quantité de substance contaminante ou par la mesure d'un marqueur biologique modifié par l'action des métaux.

L'utilisation de ces méthodes de surveillance concerne la prévention individuelle et les études épidémiologiques et quelquefois des cas médico-légaux.

La surveillance biologique (IBE) détecte l'imprégnation et les effets sur l'individu. Elle peut ainsi venir en complément d'une surveillance atmosphérique et peut confirmer les résultats des contrôles d'ambiance (VME, VLE). La figure 2 résume ces inter-relations.

Les prélèvements doivent être effectués dans des conditions rigoureuses d'hygiène. Les dosages sanguins sont effectués sur sang veineux après nettoyage de la peau éventuellement contaminée. En ce qui concerne les prélèvements urinaires, ce sont les variations de volume des urines qui affectent le plus les résultats. Les IBE pour les métaux dont l'excrétion dépend de la diurèse sont exprimés par rapport à la créatinine.

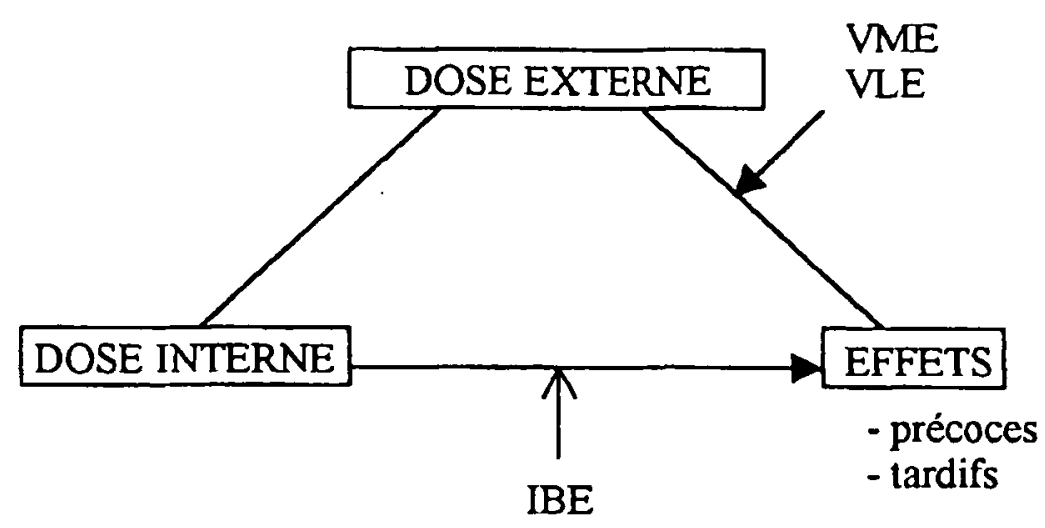

Figure 2 : Inter-relations entre dose interne et dose externe et effets. 
Le moment du prélèvement est important et dépend essentiellement de la demi-vie du paramètre analysé. En effet, la distribution et l'élimination des différents métaux sont des phénomènes cinétiques. Selon la demi-vie du contaminant étudié, on distingue : les indicateurs pour lesquels le prélèvement est effectué avant le poste (soit après $16 \mathrm{~h}$ sans exposition), pendant le poste ou en fin de poste (soit pendant les 2 dernières heures de l'exposition professionnelle et à la fin de la semaine de travail (après 5 jours de travail consécutifs). Les indicateurs pour lesquels le moment du prélèvement n'a pas d'importance concernent les substances dont les demi-vies sont très longues ; les substances s'accumulent dans l'organisme au cours des années.

Les résultats des analyses sont interprétés par comparaison à des valeurs de référence. Lors de l'interprétation des données fournies par la surveillance biologique, il faut tenir compte des différences intra- et interindividuelles quant au niveau des indicateurs biologiques pour les mêmes conditions d'exposition professionnelle (contamination ou détérioration des échantillons au cours du prélèvement, de l'analyse, condition de travail : fluctuation de l'exposition, température et humidité, état physiologique et état de santé du sujet, différence de mode de vie, environnement, ...).

Ainsi dans la plupart des cas, les IBE ne doivent être utilisés que comme valeur moyenne d'un groupe exposé et non pour un travailleur isolé (2-6).

Les techniques d'analyses des métaux sont très diversifiées : activation neutronique, fluorescence, chromatographie en phase liquide de haute performance ou en phase gazeuse : spéciation. On a le plus souvent recours à la spectrométrie d'absorption atomique et la torche à plasma couplée à la spectrométrie de masse.

1) La spectrométrie d'absorption atomique en flamme est une méthode applicable pour les milieux biologiques au calcium, lithium, zinc, cuivre et fer.

2) La spectrométrie d'absorption en four graphite est sensible et convient à la majorité des éléments. De nombreuses interférences chimiques sont réduites en utilisant une petite plaque en graphite pyrolytique (la plate-forme de l'vov) ou des additifs modificateurs.

L'effet Zeeman est efficace pour la correction des absorptions non spécifiques. Le four présente l'avantage d'une faible consommation de l'échantillon (100 à $200 \mu \mathrm{l})$, tandis que la flamme consomme généralement plusieurs millilitres par minute. Les techniques par absorption atomique peuvent faire appel à la méthode des hydrures (Se, As, $\mathrm{Hg}, \mathrm{Sn}$ ). Elle permet de sensibiliser la mesure. Un cas particulier est représenté par le $\mathrm{Hg}$ qui possède la propriété particulière de dégager une vapeur atomique stable à température ordinaire.
3) La spectrométrie d'émission optique en plasma induit (ICP-OES) est une méthode en plein essor. La méthode multi-élémentaire (dans le sérum, détermination conjointe possible par exemple d'arsenic, cadmium, zinc, plomb) présente un très large domaine de mesures et les éventuelles perturbations spectrales peuvent être minimisées par un monochromateur de haute résolution (spectrométrie de masse par plasma à couplage inductif).

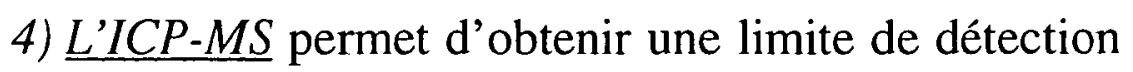
comparable à la spectrométrie d'absorption atomique tout en mélangeant les possibilités multi-élémentaires. Les applications biologiques en sont très étendues (près de $90 \%$ ) à tous les minéraux de la classification périodique du dosage simple à leur spéciation. Il peut avoir une gamme dynamique allant jusqu'à six ordres de grandeur. Il est principalement utilisé dans le domaine de l'analyse des traces et des ultra traces. (5min/échantillon pour la gamme complète des éléments traces).

5) La fluorescence $X$ est également une analyse multiélémentaire simple, rapide et sensible (intérêt pour les dosages tissulaires).

6) L'activation neutronique consiste à irradier le matériel biologique par un flux de neutrons thermiques au moyen d'un réacteur nucléaire. Seuls les laboratoires très spécialisés peuvent donc utiliser ce type de méthode. Les isotopes radioactifs obtenus sont dosés par spectrométrie gamma.

7) Spéciation : La chromatographie en phase liquide de haute performance (HPLC) ou en phase gazeuse (CPG) est utilisable en fonction des couplages, pour des dosages multi-élémentaires totaux, pour la spéciation (As, $\mathrm{Cd}, \mathrm{Cr}, \mathrm{Ni}, \ldots$ ) ou encore pour des études de biodisponibilité. Pour le type de détection, on a le plus souvent recours à la spectrométrie de masse, absorption atomique et ICP (7).

Le choix de la technique est un compromis qui fait appel à nombre de critères à classer en fonction des objectifs du laboratoire (nature de l'élément à doser, nature de l'échantillon, ...) (8).

\section{Métaux et exposition pro- fessionnelle}

\section{Plomb}

\section{Sources d'exposition}

Bien que le plomb soit un métal très répandu, les intoxications ne sont plus aussi fréquentes ni aussi graves qu'il y a quelques années.

Les applications industrielles sont nombreuses. Les 
deux principaux dangers sont représentés par les vapeurs et les poussières.

Les vapeurs sont formées dans les travaux de purification des minerais, de fonderie, le rivetage à chaud, le découpage au chalumeau de vieilles tôles peintes au minium (sesquioxyde de plomb : $\mathrm{Pb}_{3} \mathrm{O}_{4}$ ) ou à la céruse (carbonate basique de plomb $\mathrm{Pb}(\mathrm{OH})_{2}, 2 \mathrm{PbCO}$ ).

Les poussières sont d'autant plus dangereuses qu'elles sont plus fines : travaux de récupération de vieux plomb, fabrication d'accumulateurs, tréfilerie en plomb, polissage d'objets en plomb par brosse métallique rotative, ponçage à sec, destruction à la flamme de peintures à base de plomb.

Les ustensiles de cuisine, tels les couverts, récipients en étain, les récipients en faïence recouverts d'émaux mal cuits.

Les autres sources alimentaires sont variées :

- Aliments végétaux traités par herbicides à base d'arséniate de plomb

- Farine provenant de blé écrasé par des meules réparées par du plomb fondu

- Aliments grillés au feu de bois avec des bois de démolition peints avec des peintures au plomb

- Ingestion de gibiers contenant de nombreux plombs de chasse

- Enfants qui grignotent les peintures écaillées des vieilles maisons construites avant 1948

- La vie moderne, enfin, fait inhaler à l'homme, parmi les multiples polluants auxquels il ne peut se soustraire, du plomb provenant du gaz d'échappement des automobiles à essence, mais cette source est en voie de disparition.

\section{Métabolisme}

En milieu professionnel, la principale voie d'absorption (fonction de la solubilité et de la granulométrie) des dérivés minéraux du plomb est pulmonaire (environ 35 à $50 \%$ ) sous forme de vapeurs, de poussières ou de fumées : la voie digestive ne doit cependant pas être sous-estimée (5 à $10 \%$ du plomb ingéré sont absorbés chez l'adulte) : l'absorption se faisant par le biais des mains souillées, par le fait de fumer et de boire sur les lieux de travail, par l'ingestion de plomb préalablement inhalé. L'absorption par voie cutanée du plomb inorganique est faible. Dans le sang, $90 \%$ du plomb sont liés aux érythrocytes et stockés pour 80 à $90 \%$ dans les os. La fixation du plomb dans les organes de stockage provoque un phénomène d'accumulation, en particulier au niveau de l'os cortical, et détermine l'existence d'un pool de métal susceptible d'être libéré dans le sang. Ceci peut se produire plusieurs années après l'arrêt d'une exposition importante, lors d'évènements particuliers provoquant une modification de la trame osseuse (fracture, ostéoporose, ...) et au cours de diverses situations où les besoins en calcium augmentent (grossesse et allaitement) (9). Si la demi-vie du plomb dans les tissus mous et dans le sang est d'environ 30 jours, celle du plomb dans l'os est d'une vingtaine d'années. Le plomb sanguin représente $2 \%$ de la charge corporelle en plomb dans l'organisme. L'élimination fécale représente $15 \%$ du plomb ingéré, $80 \%$ du plomb absorbé sont éliminés par voie urinaire, un peu par la bile, la sueur et les phanères $(<8 \%)$.

Différentes études ont montré que le plomb traverse le placenta dès le premier trimestre et contamine le fotus (10).

\section{Mécanisme de toxicité}

La susceptibilité individuelle entre largement en ligne de compte. C'est à l'état libre ionisé que le plomb exerce ses effets toxiques sur la cellule.

Le plomb modifie la biologie de la cellule en perturbant de nombreux ions métaboliques et différents processus physiologiques. Il inhibe la biosynthèse de l'hème, aussi bien de l'hémoglobine que des cytochromes, catalyse la réaction de peroxydation des lipides et interfère avec les processus médiés par le calcium au niveau membranaire et cytosolique. Le plomb peut ainsi altérer certains mécanismes neurologiques jouant un rôle essentiel dans le développement cérébral.

Le plomb ionisé modifie les propriétés de nombreuses protéines cytosoliques et membranaires, en se liant de façon réversible avec les groupements thiol. Le plomb inhibe ainsi l'activité de nombreuses enzymes et particulièrement celle de deux enzymes de la voie de biosynthèse de l'hème, l'acide $\delta$-aminolévulinique déshydratase (ALAD) et la ferrochélatase. L'inhibition de l'ALAD s'accompagne d'une excrétion urinaire augmentée d'acide $\delta$-aminolévulinique (ALA). L'inhibition de la ferrochélatase entraîne l'accumulation de protoporphyrine érythrocytaire libre, en particulier la protoporphyrine-zinc (PPZ). Il en résulte une carence en hème, qui est le groupe prosthétique présent dans l'hémoglobine, dans certaines enzymes de la respiration cellulaire, comme les cytochromes, et dans d'autres enzymes protectrices de processus oxydatifs, comme la catalase. Cette carence en hème affecte donc non seulement la synthèse de l'hémoglobine au niveau du système hématopoïétique, mais aussi des processus cellulaires critiques au niveau de tous les tissus, comme l'activité respiratoire mitochondriale ou le métabolisme oxydatif. La carence en hème induit également l'activation de la protéine kinase $\mathrm{R}$ (PKR) conduisant à la phosphorylation de la sous-unité de l'un des facteurs d'initiation de la synthèse protéique (elF-2). Cette modification covalente inhibe très fortement la synthèse protéique. 
L'effet pro-oxydant du plomb s'exercerait principalement par l'intermédiaire de l'accumulation de l'ALA, entraînant une synthèse accrue d'ion superoxyde. Par ailleurs, les systèmes protecteurs de l'organisme contre l'oxydation sont perturbés dans les érythrocytes de sujets exposés, comme en témoigne une augmentation des concentrations en lipoperoxides, en méthémoglobine, en glutathion oxydé et en activité glutathion peroxydase. Il agit aussi en fixant le sélénium, diminuant ainsi l'activité de la glutathion peroxydase.

Le plomb interagit avec le calcium à différents niveaux cellulaires. Il inhibe les systèmes de transport membranaire comme les pompes (ATPases-Ca2 ${ }^{+}$et plus indirectement ATPase- $\mathrm{Na}^{+} / \mathrm{K}^{+}$) et certains canaux calciques. Ainsi, le plomb libre ionisé s'oppose à l'influx de calcium par les canaux voltage-dépendants, mais il ne semble pas pénétrer dans le cytosol par cette voie. Parmi ces canaux, ceux de type L (sensibles aux dihydropyridines, s'ouvrant à la suite d'une dépolarisation relativement importante et s'inactivant lentement) apparaissent les plus sensibles au plomb. Cet effet inhibiteur est rapidement réversible il est également spécifique puisque les canaux sodiques et potassiques voltage-dépendants sont insensibles au plomb. Ainsi, le plomb, en modifiant les flux de calcium, peut indirectement altérer les innombrables réactions intracellulaires dépendant des concentrations de calcium libre ionisé.

Le plomb affecte la neurotransmission glutamatergique impliquée dans la mémorisation et l'apprentissage.

Le glutamate est le principal neurotransmetteur excitateur dans le cerveau. Le plomb interfère sur la transmission neuroexcitatrice glutamatergique en agissant sur une famille de récepteurs du glutamate, les récepteurs NMDA (N-méthyl-D-asparate). Ces récepteurs sont en fait des canaux ioniques physiologiquement activés par la liaison conjointe du glutamate et de la glycine sur deux sites distincts. L'ouverture de ces canaux permet un influx important de sodium et de calcium. Les récepteurs NMDA sont impliqués au cours du développement dans la synaptogénèse et participent aux mécanismes élémentaires de mémorisation et d'apprentissage tels que la potentialisation à long terme (LTP).

Ainsi, le plomb en augmentant ou en diminuant l'efficacité de la transmission glutamatergique (selon sa concentration et celle du cotransmetteur, la glycine) pourrait provoquer des anomalies du développement du système nerveux central durant son ontogénèse et avoir des effets variables sur la toxicité induite par le glutamate. Il convient aussi de rappeler qu'à concentration plus élevée, le plomb exerce un effet toxique sur les neurones comme sur beaucoup d'autres types cellulaires par apoptose ou nécrose, indépendamment de ses capacités à moduler la transmission glutamatergique.

L'intoxication saturnine entraîne également une néphropathie tubulo-interstitielle chronique (NTI) avec, au début, une atteinte tubulaire (perte de sel, acidose tubulaire, hyperkaliémie, aminoacidurie, glucosurie) et surtout une hyperuricémie avec crise de goutte due à une augmentation de la réabsorption tubulaire de l'acide urique. Plus tard, c'est celui d'une insuffisance rénale chronique avec une protéinurie modérée, voire absente, un sédiment urinaire normal, une uricémie toujours disproportionnellement élevée par rapport au degré d'insuffisance rénale et l'hypertension artérielle est de règle, souvent sévère.

\section{Manifestations cliniques}

C'est ce qu'on a coutume d'appeler le saturnisme. Cette intoxication est d'apparition progressive et capricieuse et est influencée par de multiples facteurs.

Ses effets toxiques sur l'organisme sont hématologiques (anémie par perturbation de la biosynthèse de l'hème et diminution de la durée de vie des érythrocytes), rénaux (risques de néphropathie chronique) et neurologiques (encéphalopathie d'expression variable, mais affectant le développement intellectuel chez l'enfant).

Des effets sur le système nerveux périphérique (paralysies partielles) ou le système cardiovasculaire (hypertension artérielle), sur la reproduction et le système digestif (constipation) sont également observés (11, 12). Des évaluations sont en cours sur la cancérogénicité potentielle du plomb.

Comme pour de nombreux métaux, les catégories professionnelles exposées peuvent être prises en charge pour la pathologie en rapport avec le plomb et ses composés (13).

\section{Surveillance biologique (14)}

Le plomb est dosé par une méthode de spectrophotométrie d'absorption atomique sur le sang total $(90 \% \mathrm{du}$ plomb est intraérythrocytaire).

Certains tests sont le témoin de l'exposition : plombémie, plomburie spontanée ou provoquée, plomb osseux. D'autres sont le témoin des répercussions sur l'organisme : acide delta aminolévulinique urinaire (ALA U), protoporphyrines intraérythrocytaires (PPE) en particulier sous forme de protoporphyrines zinc (PPZ) mais aussi de protoporphyrines libres (hémoglobine, hématies à granulations basophiles, coproporphyrinurie,...). La plombémie est le meilleur indicateur d'exposition au plomb des semaines précédentes lorsque l'exposition est stable. Elle s'élève dès le début de l'exposition, elle varie en fonction des pics d'exposition pour atteindre un état d'équilibre, quelques mois après le 
début de l'exposition et diminue différemment selon le niveau d'exposition un mois après l'arrêt de l'exposition. Ce dosage nécessite une parfaite technique de prélèvement étant donné le risque de contamination de l'échantillon : les prélèvements doivent être faits en dehors des locaux de travail, sur des sujets douchés ne portant pas leurs vêtements de travail (un dosage le lundi matin est préférable). La peau doit être parfaitement lavée avant le prélèvement qui sera fait avec un dispositif d'aspiration sous vide dans un tube garanti sans plomb (bouchon compris) sur anticoagulant (EDTA ou héparine). D'un point de vue médico-légal, les dosages devront impérativement être faits par des laboratoires agréés (cf Journal Officiel, décret du $01 / 02 / 88$ et arrêt du 15/09/88), participant régulièrement à des contrôles de qualité. Dans l'interprétation des résultats, on tiendra compte des sources d'exposition extra-professionnelle liées à l'environnement (gaz d'échappement) et à l'alimentation (eau potable peu calcaire dans des conduites en plomb, aliments acides conservés dans des récipients émaillés, consommation excessive de vin) ainsi que les hobbies comme le tir.

Les valeurs d'exposition pour le plomb, concernant la population générale et les indices biologiques des personnes exposées en France, Allemagne et Etats-Unis, sont rapportées dans le Tableau II.

L'ALA urinaire est le témoin des effets sur l'organisme après une exposition récente : c'est un bon test en milieu professionnel en cas d'exposition brève ou accidentelle. Il s'élève précocement dès la deuxième semaine et est bien corrélé à la plombémie quand celle-ci atteint $600 \mu \mathrm{g} / \mathrm{L}$. La sensibilité est moyenne : elle ne s'élève que lorsque la plombémie atteint $400 \mu \mathrm{g} / \mathrm{L}$ et se normalise rapidement à l'arrêt, augmentée en cas de porphyrie aiguë, de maladie hépatique, de tyrosinémie héréditaire. Ce prélèvement est sensible à la lumière.

Les protoporphyrines érythrocytaires ou leur fraction liée au zinc (PPZ) sont des indicateurs de l'exposition des mois précédents $(95 \%$ des protoporphyrines sont liées au zinc). Cet examen est simple, rapide et de coût modéré, plus sensible que le dosage de l'ALA urinaire, indépendant de toute contamination par le plomb. Ce dosage est fortement corrélé à la plombémie entre 350 et $800 \mu \mathrm{g} / \mathrm{L}$ de plombémie. En cas d'exposition stable et prolongée, les PPZ sont de bons indicateurs de pool de plomb biologiquement actif. Les PPZ s'élèvent plus tardivement que le plomb urinaire, de 2 à 10 semaines après le début de l'exposition, dès que la plombémie atteint $200 \mu \mathrm{g} / \mathrm{L}$ et n'augmentent plus au-delà d'une plombémie de $900 \mu \mathrm{g} / \mathrm{L}$; les concentrations diminuent lentement (2 à 3 mois après l'arrêt de l'exposition). Ce dosage est peu spécifique : une carence en fer, une anémie hémolytique ou un trouble du métabolisme des porphyrines peuvent entraîner une augmentation des PPZ. Si l'analyse de la PPZ est faite par hématofluorométrie, un résultat élevé de carboxyhémoglobine entraîne une sous-estimation de la PPZ. Ce dosage en micro-méthode est possible. Les résultats devront toujours être exprimés en $\mu \mathrm{g} / \mathrm{g} \mathrm{Hb}$. Un seul tube suffit pour l'analyse de la plombémie, de l'hémoglobine et de la PPZ.

Le dosage de l'ALA déshydratase, sensible et spécifique a été proposé pour la surveillance biologique mais présente certains inconvénients : variabilité importante d'un sujet à l'autre, décomposition à la lumière, corrélation moyenne avec l'intensité de l'exposition, mise en œuvre dans les 4 heures.

Les dosages de la plomburie spontanee, des copro- et uro-porphyrines urinaires sont moins utiles. La plomburie spontanée varie en fonction de l'alimentation, de l'environnement, de l'eau de boisson et de la fonction rénale.

Les hématies à granulations basophiles sont d'un intérêt limité car sans parallélisme avec la gravité de l'intoxication et soumises à de grandes fluctuations.

Le test de plomburie provoquée par EDTA calcidisodique permet d'affirmer le diagnostic de saturnisme : c'est le meilleur indicateur biologique de la dose interne, il évalue la quantité de plomb mobilisable biologiquement actif.

A l'avenir, le dosage du plomb non invasif dans l'os par fluorescence $X$ devrait permettre une meilleure appréciation de la charge corporelle en plomb.

\section{Cadmium}

\section{Sources d'exposition}

Le cadmium entre dans la composition d'alliages avec l'acier, le zinc et le cuivre. Il permet la fabrication de batteries et d'accumulateurs rechargeables et la fabrication de revêtements anticorrosion des métaux, par électrolyse ou par trempage.

Les composés minéraux du cadmium sont des stabilisants des matières plastiques et des pigments jaunes ou oranges pour peintures, encres et émaux. Ils sont également utilisés pour la fabrication des fusibles, de cellules photoélectriques, de semi-conducteurs et de substances luminescentes.

C'est aussi un contaminant des engrais phosphatés, des boues d'incinérateur parfois utilisées comme engrais

Il faut savoir également qu'une cigarette peut apporter de 0,5 à $2 \mu$ g de cadmium.

\section{Métabolisme}

Le cadmium est faiblement absorbé au niveau du tractus digestif, environ $5 \%$ atteignent la circulation san- 


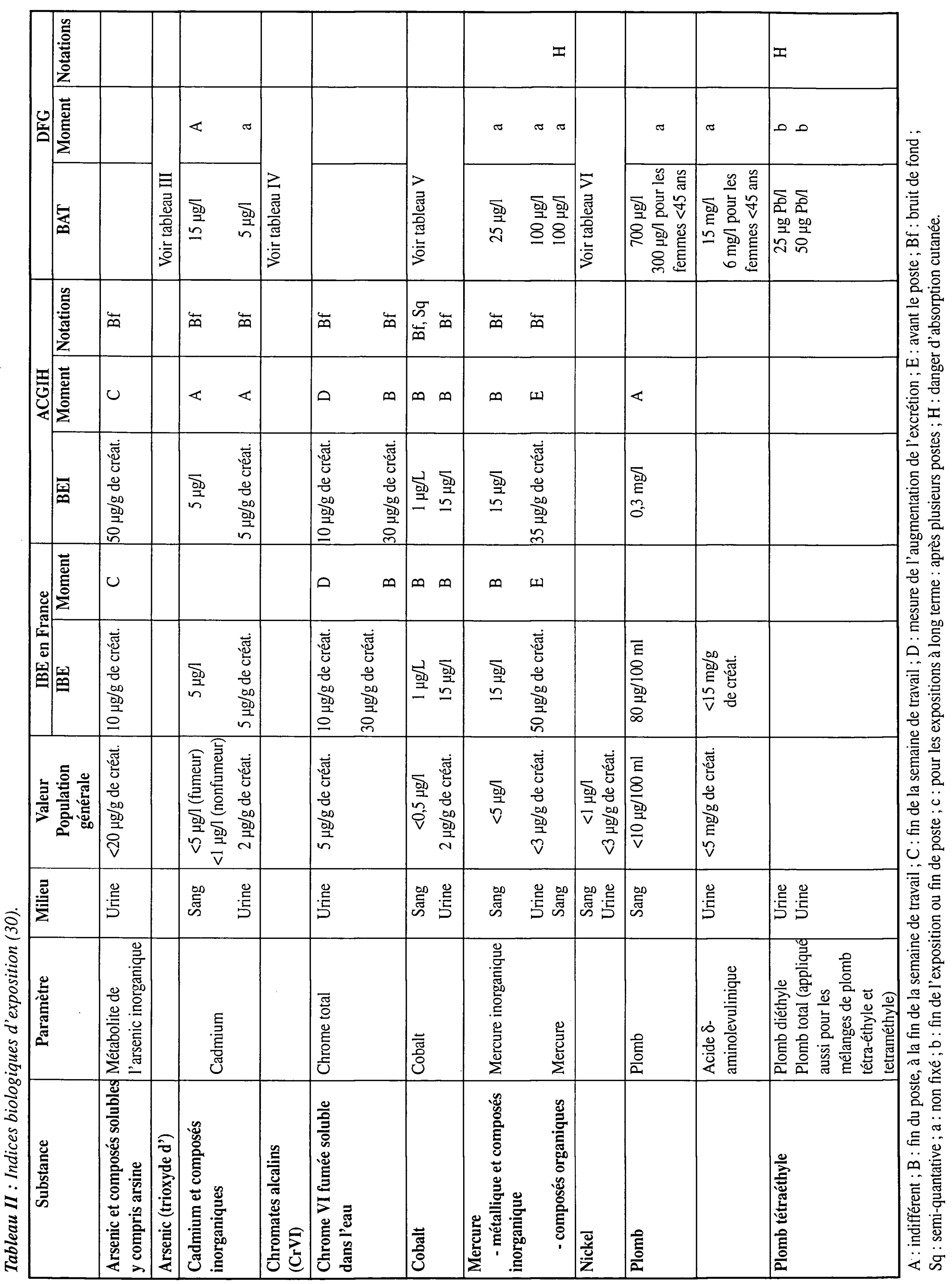


guine. Les deux principales voies d'absorption du cadmium sont pulmonaire (environ 10 à $60 \%$ ) et digestive par le biais de mains souillées. L'absorption cutanée est négligeable. La majorité $(70-80 \%)$ du cadmium sanguin est intra-érythrocytaire. Le stockage du cadmium est hépatique et rénal, fixé à la métallothionéine. La demi-vie du cadmium sanguin est de 40 à 80 jours. L'excrétion du cadmium absorbé, faible et très lente, s'effectue essentiellement par voie urinaire et très faiblement par voie fécale $(<1 \%)$ et par les phanères. Le cadmium est un toxique très cumulatif : sa demi-vie biologique varie de 10 à 30 ans.

\section{Mécanisme de toxicité}

Ce métal présente une affinité pour les groupements thiols à l'origine de nombreuses perturbations métaboliques et enzymatiques.

Le cadmium inhibe les canaux calciques et les ATPases calcium dépendantes.

Le complexe cadmium-métallothionine est toxique pour le rein.

Il est réabsorbé par endocytose au niveau du tube proximal, dégradé par les lysozomes et le cadmium libre est relâché dans la cellule tubulaire où il a une action toxique.

L'atteinte rénale est celle d'une tubulopathie proximale .

La protéinurie est de type tubulaire et il y a élimination de la $\beta 2$ microglobuline. On note une glucosurie, une amino-acidurie, une hyperphosphaturie et une acidose tubulaire. L'hyperuraturie peut entraîner une lithiase. L'hypercalciurie est fréquente, à l'origine de lithiase et surtout la fuite du calcium et du phosphore causent l'ostéoporose / ostéomalacie.

\section{Manifestations cliniques}

L'inhalation de vapeurs et de fumées à concentration modérée peut être à l'origine d'un épisode de «fièvre des métaux». De façon exceptionnelle, l'atteinte respiratoire (emphysème) peut se compliquer d'une atteinte hépatique (cytolyse) et rénale (tubulopathie proximale avec diabète phospho-gluco-aminé) et une atteinte osseuse (ostéomalacie, parfois ostéoporose, fractures spontanées, fissures osseuses,...).

Comme pour de nombreux métaux, les catégories professionnelles exposées peuvent être prises en charge pour la pathologie en rapport avec le cadmium et ses composés (15).

\section{Surveillance biologique (14)}

Le dosage du cadmium sanguin est un indicateur d'imprégnation récente des 3-4 derniers mois mais aussi de la charge corporelle. Lors de la première année d'exposition ou quand l'exposition est fluctuante, le dosage sanguin est à privilégier. Il augmente pendant 4 à 6 mois puis se stabilise en plateau : en cas d'exposition faible (quelques $\mu \mathrm{g} / \mathrm{m}^{3}$ ), le taux sanguin traduit l'exposition récente. Le tabagisme et l'alimentation peuvent gêner l'interprétation des résultats. Les concentrations augmentent avec l'âge. Le cadmium sanguin est moins bien corrélé à l'intensité de l'exposition que le cadmium urinaire.

Les valeurs d'exposition pour le cadmium, concernant la population générale et les indices biologiques des personnes exposées en France, Allemagne et ÉtatsUnis, sont rapportées dans le Tableau II.

Le cadmium urinaire reflète surtout l'exposition chronique et la charge corporelle. C'est le premier indicateur à utiliser dans la gestion du risque à long terme. Dès que les sites de stockage sont saturés, la fonction tubulaire rénale est atteinte, elle entraîne une augmentation de l'excrétion urinaire du cadmium, une bonne corrélation existe entre les taux de cadmium urinaire, l'intensité de l'exposition et le degré d'atteinte rénale : pour un taux de cadmium urinaire proche de $20 \mu \mathrm{g} / \mathrm{g}$ de créatinine, des effets irréversibles tubulaires rénaux sont constatés : pour un taux de cadmium urinaire voisin de $4 \mu \mathrm{g} / \mathrm{g}$ de créatinine, des effets cytotoxiques ( $\beta 2$ microglobulinurie) apparaissent. Pour un taux de cadmium urinaire entre 10 et $20 \mu \mathrm{g} / \mathrm{g}$ de créatinine et si les taux de $\beta 2$ microglobulinurie et de rétinol binding protein sont inférieurs à $300 \mu \mathrm{g} / \mathrm{g}$ de créatinine, le risque de développer une atteinte tubulaire rénale à l'arrêt de l'exposition est faible. Le tabac, l'alimentation, l'âge, le moment du prélèvement dans la journée (variations circadiennes : taux maximum le matin) modifient faiblement les taux. Au total, le cadmium urinaire doit être utilisé comme premier indicateur biologique d'exposition, systématiquement associé à d'autres indicateurs biologiques d'effet (cadmium sanguin, indicateurs d'atteinte tubulaire : $\beta 2$ microglobulinurie (valeur normale $<300 \mu \mathrm{g} / \mathrm{g}$ de créatinine), rétinol binding protein (valeur normale $<300 \mu \mathrm{g} / \mathrm{g}$ de créatinine), indicateur d'atteinte glomérulaire : albuminurie (valeur normale $<15 \mu \mathrm{g} / \mathrm{g}$ de créatinine) dès que le cadmium urinaire est supérieur à $5 \mu \mathrm{g} / \mathrm{g}$ de créatinine. Le dosage isolé du cadmium sanguin ne permet pas d'assurer une prévention efficace.

Pour les dosages urinaires et sanguins il est nécessaire de se prémunir d'une contamination externe en se lavant les mains après enlèvement des habits contaminés et en utilisant des récipients et des réactifs adéquats exempts de cadmium et en réalisant le prélèvement le matin avant l'arrivée au travail.

\section{Arsenic}

\section{Sources d'exposition}

L'arsenic est présent comme impureté dans de nombreux minerais. Les principales circonstances d'exposi- 
tion professionnelle sont le grillage de minerais arsenicaux (fonderies de cuivre surtout, mais aussi d'or, de plomb et de zinc), la fabrication des organo-arsenicaux, le traitement du bois par les fongicides, la verrerie et la cristallerie (affinage du verre), la tannerie, la fabrication de pesticides et le traitement de la vigne.

L'arsenic sous forme de trioxyde, a été utilisé en thérapeutique médicale sous forme de liqueur de Fowler dans le traitement de certaines dermatoses, notamment du psoriasis. L'arsenic est aussi présent dans la fumée de cigarette. Il se trouve aussi dans l'eau de surface ou souterraine de certaines régions; ce métal se retrouve ainsi présent un peu partout dans notre environnement, que ce soit dans l'air, dans l'eau, dans le sol et même dans la nourriture. Une contamination des sols et des eaux a été observée à proximité des mines d'or (Salsigne,...).

L'hydrogène arsénié ou arsine est un gaz incolore plus lourd que l'air. La pénétration dans l'organisme est exclusivement respiratoire. C'est le plus toxique des dérivés de l'arsenic.

Hormis quelques applications dans l'industrie électronique comme gaz de dopage dans la fabrication de semi-conducteurs et en synthèse organique, le plus souvent la formation d'hydrogène arsénié est accidentelle.

Les principales circonstances de dégagement accidentel se rencontrent dans la métallurgie: traitement de minerais ayant de l'arsenic comme impureté (zinc, cuivre, étain, plomb et cobalt), détartrage chimique acide de chaudières, bronzage d'art, restauration de peintures contenant des dérivés arsenicaux. Ce risque existe chaque fois que l'arsenic est en contact avec de l'hydrogène naissant ou des solutions acides.

\section{Métabolisme}

L'absorption dépend de la solubilité, de la granulométrie et de la valence de l'arsenic Elle peut se faire par voie digestive pour $80 \%$ environ de la quantité ingérée (par le biais de mains souillées pour l'arsenic inorganique, par le biais de l'alimentation ou de pesticides pour l'arsenic organique), par voie pulmonaire surtout en milieu professionnel pour les poussières et les vapeurs et faiblement par voie cutanée. Il est rapidement distribué dans l'organisme et se fixe aux protéines, pour s'accumuler dans le foie, les muscles, la peau. Les composés trivalents sont transformés au niveau du foie en composés pentavalents. La demi-vie sanguine est de 7 jours. Environ $70 \%$ des composés inorganiques absorbés sont éliminés rapidement dans les urines $(50 \%$ dans les deux jours ; $90 \%$ en six jours) sous forme de dérivés méthylés : acide monométhylarsonique (ou dérivé monométhylé (MMA)) pour $25 \%$ et acide diméthylarsonique (ou dérivé dimé- thylé (DMA)) pour $50 \%$ et sous forme inchangée pour $25 \%$. Il existe des variations individuelles du métabolisme. La demi-vie des métabolites urinaires est de 2 à 6 jours. L'élimination s'effectue aussi par la bile et par les phanères (poils, cheveux). L'arsenic s'accumule dans les phanères permettant un diagnostic rétrospectif. L'arsenic alimentaire est éliminé pour la majeure partie sous forme inchangée dans les urines et ne présente pas de toxicité.

\section{Mécanisme de toxicité}

L'action toxique s'exerce en bloquant les groupements thiols. Il se lie immédiatement aux protéines. L'affinité des composés arsenicaux pour les protéines tissulaires rend compte de la disparition rapide de l'arsenic du sang circulant. Les arseniates sont aussi des anti-métabolites des phosphates. Ils sont mutagènes et cancérogènes.

Il y a peut-être un phénomène de tolérance (mithridatisation) ; l'exemple le plus connu est celui des arsenicophages du thyrol qui pouvaient apparemment ingérer jusqu'à $1 \mathrm{mg}$ d'arsenic par jour.

L'arsenic perturbe aussi la phosphorylation oxydative

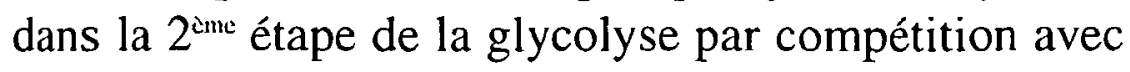
les ions phosphates au niveau de la réaction de la glycéraldéhyde déshydrogénase.

L'arsenic se fixe sur le glycéraldéhyde 3 phosphate et cela a pour conséquence la non production d'ATP (figure 3).

\section{Manifestations cliniques}

L'intoxication chronique se manifeste par des atteintes cutanées (mélanodermie, kératodermie, maladie de Bowen) des atteintes des ongles, des cheveux et des muqueuses. Une polyneuropathie, ainsi que des troubles hépatiques et rénaux importants, sont signalés. L'intoxication chronique par ingestion d'arsenic induit une fibrose portale. L'hypertension portale de type présinusoïdale est importante et peut conduire à des hémorragies digestives par rupture de varices œsophagiennes. La responsabilité de l'arsenic dans la survenue de cirrhose a été soulevée dans certains groupes professionnels, notamment les vignerons.

L'ingestion chronique d'arsenic peut aussi être à l'origine d'angiosarcomes et d'hémangio-endothéliomes. L'angiosarcome du foie est répertorié comme maladie professionnelle au titre du tableau $n^{\circ} \underline{20} \mathrm{D}$ du R.G. et $n^{\circ} 10$ du R.A. Le délai de prise en charge est de 40 ans. L'hydrogène arsénié provoque une hémolyse intra-vasculaire massive avec anémie et hémoglobinurie.

Comme pour de nombreux métaux, les catégories professionnelles exposées peuvent être prises en charge pour la pathologie en rapport avec l'arsenic et ses composés (16). 


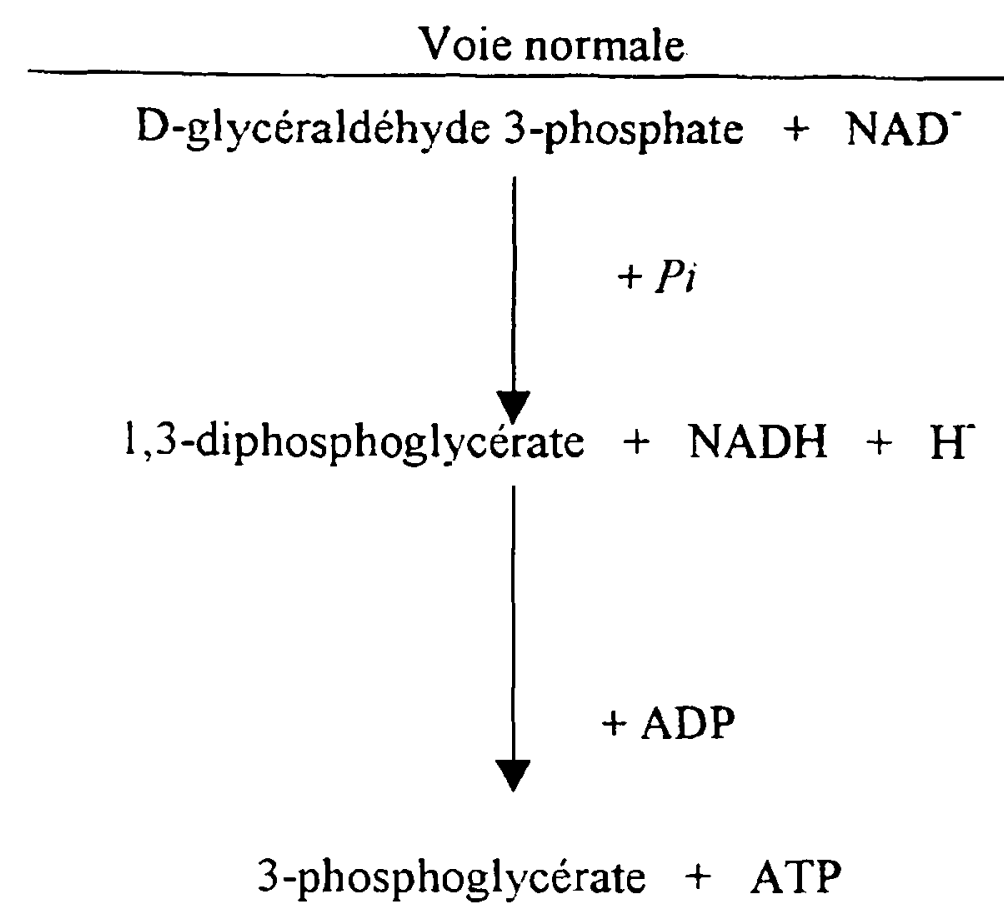

Présence d'arsenic

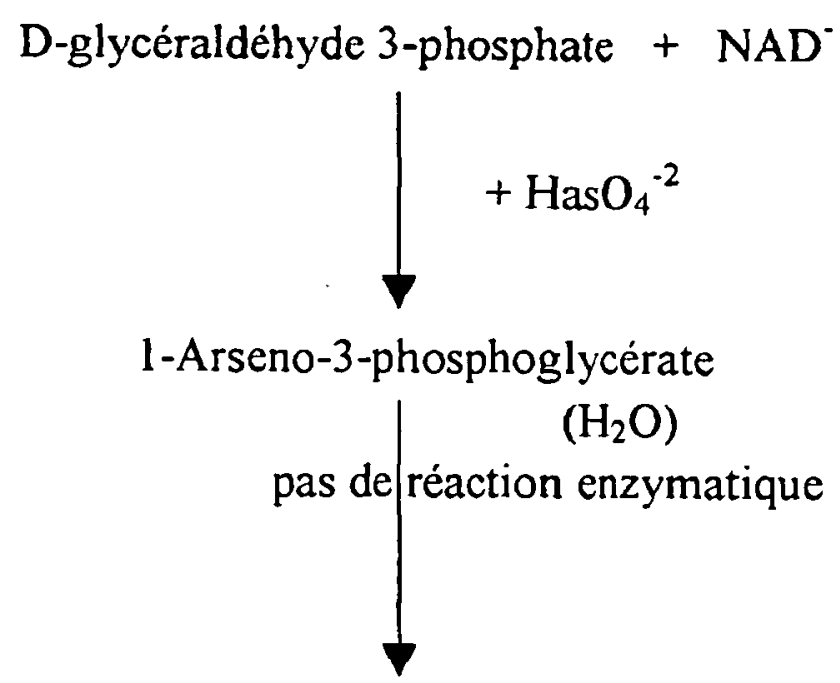

3-phosphoglycérate $+\mathrm{HAsO}_{4}^{-2}$

(ATP non produit)

Figure 3 : Liaison de l'arsenic avec le glycéraldéhyde 3-phosphate et inhibition de la production d'ATP.

\section{Surveillance biologique}

Le dosage de l'arsenic sanguin est peu utilisé en milieu professionnel (en dehors des situations d'intoxication aiguë). Il est le reflet de l'exposition récente ; sa corrélation avec l'intensité de l'exposition n'est pas clairement établie.

Les dosages d'arsenic urinaire en fin de poste et fin de semaine de travail sont très utiles pour la surveillance biologique de l'exposition. Les valeurs d'exposition pour l'arsenic, concernant la population générale et les indices biologiques des personnes exposées en France, Allemagne et États-Unis, sont rapportées dans le Tableau II.

Seuls les dosages d'arsenic inorganique et des dérivés méthylés doivent être utilisés pour ne pas prendre en considération l'arsenic organique d'origine alimentaire non toxique (poissons, crustacés,...) et reflètent l'exposition de la semaine aux composés inorganiques de l'arsenic et à l'arsine. Il est cependant utile de noter la consommation alimentaire des 48 heures précédant le prélèvement. Ce dernier ensemble (arsenic inorganique + MMA + DMA) est bien corrélé à l'intensité de l'exposition. Le dosage de l'arsenic urinaire ne s'applique pas à l'évaluation de l'exposition à l'arséniure de gallium, car ce composé est pratiquement insoluble (Tableau II). Des études ont montré que la spéciation de l'arsenic était un facteur important, contrôlant la mobilité et la toxicité de l'arsenic $(17,18,19)$. L'arsenic est une substance cancérogène, une corrélation a pu être établie entre les concentrations dans l'air et les valeurs retrouvées dans les urines des sujets exposés, notamment en ce qui concerne le trioxyde d'arsenic (Tableau III).
Les dosages dans les phanères (cheveux, ongles) ne sont pas recommandés en raison des grandes variations interindividuelles et de la possibilité de contamination, sauf dans le domaine médico-légal.

\section{Mercure}

\section{Source d'exposition}

Le mercure est un métal liquide à la température ordinaire ; il est très utilisé dans l'industrie sous sa forme métallique ou sous forme de dérivés inorganiques ou organiques. Le mercure métallique est utilisé dans des appareils scientifiques de précision (thermomètres, manomètres, etc.) dans l'industrie électrique, dans les processus de production de la soude et du chlore, dans la préparation d'amalgames (notamment en dentisterie), dans l'extraction de l'or à partir de ses minerais (forte pollution en Guyane due aux orpailleurs). Le mercure est utilisé dans l'industrie de la peinture, des pesticides et des médicaments.

Les dérivés inorganiques du mercure (chlorure mercurique, nitrate de mercure, fulminate de mercure, oxyde rouge de mercure) ont encore des utilisations industrielles ou pharmaceutiques.

Tableau III : Arsenic (trioxyde d') (30).

\begin{tabular}{|c|c|}
\hline $\begin{array}{c}\text { Concentration d'arsenic dans l'air } \\
\left(\mathrm{mg} / \mathrm{m}^{3}\right)\end{array}$ & $\begin{array}{c}\text { Arsenic dans l'urine } \\
(\mu \mathrm{g} / \mathrm{l})\end{array}$ \\
\hline 0,01 & 50 \\
\hline 0,05 & 90 \\
\hline $\mathbf{0 , 1 0}$ & $\mathbf{1 3 0}$ \\
\hline
\end{tabular}


Les dérivés organiques du mercure (essentiellement les alkylés tels le diméthylmercure, le chlorure d'éthylmercure ou les iodures et nitrate de méthylmercure) sont utilisés comme fongicides et dans des domaines industriels aussi variés que le papier, les cosmétiques, les matières plastiques et les peintures, mais ces utilisations décroissent régulièrement.

Dans l'environnement, les dérivés inorganiques peuvent être méthylés sous forme de méthylmercure (CH3$\mathrm{Hg}^{+}$) qui peut s'accumuler fortement dans les chaînes alimentaires, surtout aquifères.

\section{Mécanismes de toxicité}

\section{Mercure minéral}

Le mercure, comme le plomb et le cadmium, est un élément cumulatif dont la toxicité à long terme s'exerce essentiellement au niveau du système nerveux et du rein. Le mercure sous forme ionisée est un agent thioloprive puissant perturbant le fonctionnement cellulaire. Il affecte divers systèmes enzymatiques : diminution de la production énergétique cellulaire ; il est responsable d'atteintes multiples au niveau des cellules rénales et cérébrales. Il affecte également les membranes cellulaires et les acides nucléiques.

\section{Mercure organique}

Le méthylmercure, est doté d'une toxicité cumulative redoutable en raison de sa grande liposolubilité. Il se transforme à partir des ions mercure qui subissent une méthylation enzymatique ou non dans des divers systèmes, bactériens ou d'autres organismes vivant dans les eaux.

\section{Métabolisme}

Il y a lieu de distinguer le mercure métallique, métal volatil dont l'inhalation représente la principale voie d'absorption en milieu industriel, les dérivés minéraux du mercure sous l'angle de la pénétration cutanée ou de l'ingestion éventuelle, enfin, le cas du méthylmercure.

En milieu professionnel, la principale voie d'entrée du mercure métal dans l'organisme est pulmonaire (vapeurs et poussières) dont $80 \%$ sont absorbés.

L'absorption digestive du mercure métal est négligeable (inférieure à 0,01\%), l'absorption cutanée est possible. Certains sels inorganiques de mercure tel le chlorure mercurique sont très solubles et jusqu'à $10 \%$ de ces sels peuvent être absorbés par voie digestive. Les effets toxiques de toutes les formes de mercure inorganique sont liés à la formation de mercure ionisé. Le passage dans le sang et la distribution du mercure sont très rapides (en quelques minutes après le début de l'exposition). Le mercure s'accumule rapidement dans les érythrocytes mais aussi dans les reins, le système nerveux central et le foie, là, il subit une oxydation pour se transformer en ion mercureux, puis mercurique toxique. Pour le mercure métallique, le cerveau représente cependant l'organe critique en tant qu'organe cible principal de l'exposition chronique aux vapeurs de mercure. Pour les dérivés minéraux du mercure, outre les hématies, ces ions se fixent également sur l'albumine plasmatique, à parties égales entre les deux milieux. La forme $\mathrm{Hg}_{2}{ }^{+}$est thioloprive. Le lieu de stockage préférentiel est le rein qui représente environ $90 \%$ des réserves ; la contribution des autres organes est peu importante : cerveau, foie, muscles, os, phanères. La demi-vie d'élimination du mercure sanguin est biphasique (de 3 et 30 jours, respectivement). Le mercure inorganique est excrété par voie rénale et fécale, faiblement par la sueur, la salive et les phanères. Le pic d'élimination urinaire apparaît 2 à 3 semaines après l'exposition avec un plateau atteint entre 10 jours et 6 mois, la demi-vie d'élimination urinaire est de 40 à 60 jours.

Les dérivés organiques du mercure (ex : méthylmercure) sont absorbés par les voies digestive (pour plus de $90 \%$ ) mais aussi pulmonaire et cutanée. Ils se distribuent en 3 jours environ dans tout l'organisme et surtout dans le système nerveux central. Ils sont éliminés par voies biliaire et fécale (demi-vie d'élimination d'environ 70 jours) et faiblement par les urines. Une partie des dérivés organiques subit un cycle entéro-hépatique. Ils passent la barrière placentaire.

\section{Manifestations cliniques}

L'intoxication chronique évolue de manière insidieuse, l'atteinte du système nerveux central et périphérique est la plus grave : tremblements, paresthésies, éréthisme, ataxie, troubles du caractère et des performances psychomotrices. Une gingivite, une stomatite, une atteinte rénale, une atteinte oculaire peuvent être observées.

L'intoxication par les organomercuriels, en dehors de l'action tératogène chez le fœtus, provoque chez l'adulte, après plusieurs mois de latence, un ensemble de symptômes graves : ataxie cérébelleuse, mouvements choréiformes, cécité, diarrhées, vomissements, fatigue, fièvre.

Comme pour de nombreux métaux, les catégories professionnelles exposées peuvent être prises en charge pour la pathologie en rapport avec le mercure et ses composés (20).

\section{Surveillance biologique}

Le dosage du mercure inorganique sanguin total en fin de semaine et fin de poste de travail est un bon indicateur de l'exposition au mercure de la semaine précédente et est bien corrélé au taux atmosphérique. L'indice biologique d'exposition proposé devrait protéger des effets sur la santé : pour une exposition à $0,05 \mathrm{mg} / \mathrm{m}^{3}$ les concentrations sanguines de mercure sont de l'ordre 
de $25 \mu \mathrm{l}$. Ces concentrations, maximales le jour même de l'exposition, seraient bien corrélées aux symptômes avec des signes neurologiques apparaissant pour des concentrations plasmatiques supérieures à $200 \mu \mathrm{g} / \mathrm{L}$. Ce dosage étant délicat, il est important de faire appel à des laboratoires participant à des contrôles de qualité. La contamination lors du recueil du prélèvement doit être évitée.

Le dosage du mercure inorganique total urinaire permet d'apprécier l'exposition ancienne (de plus de 3 mois) au mercure inorganique et est bien corrélé à l'intensité de l'exposition. Ce paramètre est le mieux corrélé aux effets sur la santé. Pour des concentrations urinaires inférieures à $50 \mu \mathrm{g} / \mathrm{g}$ de créatinine il n'y a en général pas d'anomalies physiologiques, psychologiques ou biochimiques. Il existe de grandes variations journalières des taux de mercure urinaire plus élevés le matin. Les amalgames dentaires au mercure et certains désinfectants locaux sont une des principales sources de mercure inorganique, susceptibles d'entraîner une élévation des concentrations urinaires. Les valeurs d'exposition pour le mercure, concernant la population générale et les indices biologiques des personnes exposées en France, Allemagne et États-Unis, sont rapportées dans le Tableau II.

Au total, pour une exposition ancienne et constante, le dosage du mercure urinaire est à privilégier ; pour une exposition fluctuante, le dosage du mercure sanguin est préférable. Le dosage du mercure urinaire ne devrait être utilisé qu'après 6 mois d'exposition puisque les concentrations urinaires de mercure atteignent un état d'équilibre en 10 jours à 6 mois ; ce paramètre ne s'applique pas à l'exposition aux dérivés organiques du mercure. La spéciation du mercure émis dans l'atmosphère est importante car le $\mathrm{Hg}^{+}$se dépose plus rapidement que le $\mathrm{Hg}^{0}$ (21).

\section{CHROME}

\section{Source d'exposition}

Le chrome trouve de nombreuses applications industrielles : les chromates, le mordançage des tissus, les pigments (industrie des peintures), le tannage des cuirs, la métallurgie (ferrochrome, les aciers au chrome, les alliages avec $\mathrm{Co}, \mathrm{Mn}$ et $\mathrm{Ni}$ ), le chromage, les conservateurs du bois, fongicides.

Le soudage d'aciers au chrome peut être à l'origine de la formation d'aérosols riches en $\mathrm{Cr}$ (VI), surtout avec le procédé à la baguette enrobée.

\section{Métabolisme}

L'absorption du chrome est fonction de sa valence et de sa solubilité. Le chrome (III) est peu absorbé. Il pénètre difficilement au travers des membranes.
Le chrome hexavalent pénètre dans l'organisme par toutes les voies, principalement respiratoires (surtout pour le chrome (VI) soluble) mais aussi digestive (défaut d'hygiène) et un peu cutanée. Il se distribue principalement dans le foie, les reins, la rate et les poumons. Le chrome hexavalent peut pénétrer dans les hématies ; après réduction en chrome trivalent, il y est retenu pendant toute la durée de vie des globules rouges. Son excrétion est essentiellement urinaire sous forme de chrome (III) (> $80 \%$ ) et faiblement fécale. L'élimination urinaire est triphasique (demi-vies d'élimination : 7 heures, 15 à 30 jours, 4 ans). Il y a accumulation du chrome au cours de la semaine et tout au long de l'année chez les sujets les plus exposés (22).

\section{Mécanisme de toxicité}

Les sels de chrome (III) ont la propriété de se combiner aux protéines de la peau après pénétration par les glandes sudoripares, en formant des complexes stables, ce qui n'est pas le cas du Cr (IV) qui traverse facilement la peau, peut diffuser dans l'organisme et franchir les membranes cellulaires. Les acides aminés soufrés intracellulaires réduisent le $\mathrm{Cr}$ (IV) en $\mathrm{Cr}$ (III) et ce dernier peut alors former des complexes protéiques allergéniques.

\section{Manifestations cliniques}

Les intoxications chroniques par contact cutané ou inhalation entraînent des ulcérations locales et dermatites allergiques (douleurs et prurit, pigeonneaux, gâle du ciment, eczémas, dermites des ménagères).

Des cancers du poumon et du larynx ont été rapportés uniquement avec les dérivés hexavalents (23).

\section{Surveillance biologique}

Le dosage du chrome intraérythrocytaire serait le reflet de l'exposition au chrome (VI) (forme potentiellement la plus toxique du chrome).

Le dosage du chrome plasmatique refléterait essentiellement l'exposition à toutes les valences du chrome. Une bonne corrélation existe entre les taux de chrome urinaire, plasmatique et intra-érythrocytaire. Les taux de chrome urinaire seraient égaux à 4 fois les taux de chrome plasmatique, ce qui en fait le milieu choisi pour la surveillance en milieu professionnel.

La chromurie, prélèvement fait en fin de poste de travail (recueil des urines des 2 dernières heures du poste) est le meilleur indicateur d'exposition récente au chrome. En cas d'exposition importance, le chrome urinaire reflète l'exposition chronique mais aussi celles de la journée et de la veille. Des prélèvements en début et fin de poste permettent une bonne évaluation de l'exposition de la journée. Les valeurs des indicateurs biologiques d'exposition ne sont pas applicables au traite- 
ment de surface ; elles ont été établies à partir d'expositions aux fumées de soudage sur acier inox (exposition au chrome (VI) soluble). Pour le traitement de surface, une valeur de $5 \mu \mathrm{g} / \mathrm{L}$ du chrome urinaire en fin de poste a été proposée comme valeur de référence pour une population exposée. Pour les nouveaux travailleurs, les valeurs proposées pour le chrome urinaire sont de $7 \mu \mathrm{g} / \mathrm{g}$ de créatinine (en fin de poste) et $5 \mu \mathrm{g} / \mathrm{g}$ de créatinine (augmentation au cours du poste). Les valeurs d'exposition pour le chrome, concernant la population générale et les indices biologiques des personnes exposées en France, Allemagne et États-Unis, sont rapportées dans le Tableau II. Les chromates alcalins sont des substances cancérogènes, une corrélation a pu être établie entre les concentrations dans l'air et les valeurs retrouvées dans les urines des sujets exposés (Tableau IV).

L'influence du tabac, de l'hygiène personnelle, des modes de travail et surtout de la valence, de la solubilité et de la forme physique du chrome sont à prendre en compte. Il n'y a pas de corrélation entre les taux de chrome urinaire et les risques d'atteinte rénale. Même après plusieurs mois d'arrêt d'exposition, la chromurie peut rester supérieure aux valeurs de la population générale.

Tableau IV: Chromates alcalins (CrVI) (30).

\begin{tabular}{|c|c|c|}
\hline \multirow{2}{*}{$\begin{array}{c}\text { Concentration } \\
\text { de } \mathrm{CrO}_{3} \text { dans l'air } \\
\left(\mathrm{mg} / \mathrm{m}^{3}\right)\end{array}$} & $\begin{array}{c}|c| \\
\text { Dans les érythrocytes } \\
\text { Moment de prélèv. : c } \\
(\mu \mathrm{g} / \mathrm{l})\end{array}$ & $\begin{array}{c}\text { Dans l'urine } \\
\text { Moment de prélèv. : } \mathrm{b} \\
(\mu \mathrm{g} / \mathrm{l})\end{array}$ \\
\hline 0,03 & 9 & 12 \\
\hline $\mathbf{0 , 0 5}$ & $\mathbf{1 7}$ & $\mathbf{2 0}$ \\
\hline 0,08 & 25 & 30 \\
\hline 0,10 & 35 & 40 \\
\hline
\end{tabular}

\section{COBALT}

\section{Sources d'exposition}

Le cobalt est un métal utilisé dans diverses industries : fabrication d'alliages (aimants), industrie des métaux durs (Co-carbure de tungstène), industrie de la céramique et du verre, polissage de diamants, meulage de prothèses chirurgicales et dentaires, peintures....

\section{Métabolisme}

L'absorption dépend de la solubilité (l'oxyde de cobalt est insoluble dans l'eau et l'acide chlorhydrique, mais soluble dans l'acide sulfurique ; le sulfate de cobalt est soluble dans l'eau) et de la granulométrie des poussières de cobalt. La voie de pénétration principale en milieu industriel est pulmonaire (lente pour les oxydes) ; l'absorption cutanée est possible et l'absorption digestive est variable ( 7 à $40 \%$ ). Le cobalt est stocké dans le foie et les reins, puis est rapidement éliminé dans les urines (pour $85 \%$ ) et les selles. L'élimination débute 3 jours après le début de l'exposition. La demi-vie d'élimination est biphasique : quelques jours et 2 ans. Il y a accumulation au cours de la semaine et tout au long de l'année chez les plus exposés.

\section{Manifestations cliniques}

L'inhalation ou l'ingestion de cobalt induit un certain nombre d'effets toxiques chez l'homme. Le cobalt joue un rôle primordial dans l'apparition de pathologies pulmonaires comme la fibrose due aux métaux durs après exposition de longues durées aux poussières métalliques (24). Le carbure de tungstène aggrave les effets du cobalt.

Les manifestations de type irritatif du tractus respiratoire sont caractérisées par une toux productrice, des manifestions cutanées (eczémas de contact), des troubles hématologiques, des troubles cardiaques et des effets sur les systèmes enzymatiques (énergétique, rénal,...) sont aussi observées (25).

\section{Surveillance biologique}

Le dosage de la cobalturie en fin de journée et fin de semaine de travail, serait un bon reflet de l'exposition de la semaine précédente (si la fonction rénale est normale), bien corrélée à l'intensité de l'exposition au cobalt et à ses sels. Par contre pour une exposition aux oxydes de cobalt il n'y a pas de bonne corrélation entre l'exposition récente, le cobalt sanguin et urinaire bien que les concentrations de cobalt urinaire soient plus élevées que chez les sujets non exposés. Des prélèvements urinaires réalisés le lundi matin seraient le reflet de l'exposition à long terme. Les valeurs d'exposition pour le cobalt, concernant la population générale et les indices biologiques des personnes exposées en France, Allemagne et États-Unis, sont rapportées dans le Tableau II.

Le dosage du cobalt sanguin en fin de poste et fin de semaine de travail est le reflet de l'exposition récente au cobalt et à ses composés inorganiques. Une bonne corrélation existe entre les concentrations sanguines et urinaires de cobalt et les concentrations atmosphériques lors d'exposition récente au cobalt, à ses sels et aux métaux durs (mais pas aux oxydes). Le cobalt est une substance cancérogène, une corrélation a pu être établie entre les concentrations dans l'air et les valeurs retrouvées dans les urines des sujets exposés (Tableau $\mathrm{V}$ ). 
Tableau V : Cobalt et ses composés (30).

\begin{tabular}{|c|c|c|}
\hline \multirow{2}{*}{$\begin{array}{c}\text { Concentration de } \\
\begin{array}{c}\text { Cobalt dans l'air } \\
\left(\mathrm{mg} / \mathrm{m}^{3}\right)\end{array}\end{array}$} & \begin{tabular}{c}
$|c|$ \\
\cline { 2 - 3 }
\end{tabular} & $\begin{array}{c}\text { Cobalt } \\
(\mu \mathrm{g} / \mathrm{l}) \\
\text { Moment de prélèvement }: \mathrm{a}\end{array}$ \\
\hline 0,05 & 2,5 & $\begin{array}{c}\text { Dans l'urine } \\
(\mu \mathrm{g} / \mathrm{l})\end{array}$ \\
\hline $\mathbf{0 , 1 0}$ & $\mathbf{5 , 0}$ & 30 \\
\hline 0,50 & 25,0 & $\mathbf{6 0}$ \\
\hline
\end{tabular}

\section{NICKEL}

\section{Sources d'exposition}

L'utilisation du nickel dans les industries métallurgiques, dans la protection des métaux (nickelage) peut être la cause de certaines intoxications.

Il est utilisé dans la fabrication d'alliages, d'aciers spéciaux, de batteries ( $\mathrm{Ni}-\mathrm{Cd})$, de pièces de monnaies ustensiles de cuisine, bijoux fantaisie, comme agent mordant en teinturerie et comme pigments pour les émaux, céramique et porcelaine. Les soudeurs d'aciers au nickel sont également exposés.

\section{Métabolisme}

La pénétration du nickel est essentiellement pulmonaire pour les dérivés solubles et le gaz nickel carbonyle (l'absorption pulmonaire des composés du nickel dépend essentiellement des propriétés physico-chimiques des particules, elle est en moyenne de $20 \%$ ). L'absorption digestive est faible (10\%), l'absorption percutanée est possible (elle dépend de la nature du sel de nickel et augmente avec la sudation). Transporté, lié aux protéines (demi-vie plasmatique de 20 à 35 heures), il se distribue dans les reins et les poumons puis il est éliminé rapidement dans les fèces (pour $90 \%$ environ lorsqu'il est ingéré), dans les urines à des taux variables et faiblement dans la sueur et les phanères. La demi-vie d'élimination est de quelques heures (20 à 40 heures) pour les composés solubles et de plusieurs semaines à années pour les composés insolubles. Il existe une accumulation du nickel dans l'organisme au cours de la semaine.

\section{Manifestations cliniques}

Les sels de nickel peuvent occasionner une dermite allergique (eczéma).Les allergies respiratoires sont rares, cependant des manifestations de bronchites chroniques, de rhinites et de sinusites peuvent être observées chez des travailleurs exposés à des poussières à base de nickel. Certains composés peu solubles tels l'oxyde et le sous-sulfure de nickel ont été à l'origine de cancers des fosses nasales et du poumon ; les améliorations des conditions d'hygiène dans les usines de production ont pratiquement supprimé le risque naso-sinusien.

Le nickel-carbonyle, produit intermédiaire du raffinage du nickel, très volatil, peut entraîner des manifestations toxiques aiguës lors de l'exposition aux vapeurs : céphalées, vertiges, nausées, vomissements, œdème pulmonaire, cyanose, etc. Des hémorragies pulmonaires, cérébrales et méningées peuvent survenir et entraîner la mort. Après absorption par voie pulmonaire, il se décompose rapidement en nickel et en oxyde de carbone (26).

\section{Surveillance biologique}

Le dosage du nickel dans les urines des 8 heures (ou à défaut dans les urines de fin de poste) en fin de semaine de travail peut être utile pour la surveillance biologique et reflète l'exposition de la journée et la quantité accumulée dans l'organisme. Ces taux sont bien corrélés aux concentrations atmosphériques lors d'exposition aux composés solubles, cependant les variations circadiennes sont importantes. On se méfiera d'une contamination de l'échantillon. Lors d'exposition aux dérivés faiblement solubles (sulfure, carbonate, oxyde de nickel), l'augmentation de la concentration urinaire de nickel est plus lente et plus prolongée. Une concentration urinaire de $10 \mu \mathrm{g} / \mathrm{L}$ correspond à une exposition à $0,1 \mathrm{mg} / \mathrm{m}^{3}$ de nickel soluble. Le nickel est une substance cancérogène, une corrélation a pu être établie entre les concentrations dans l'air et les valeurs retrouvées dans les urines des sujets exposés (Tableau VI).

Les taux de nickel sanguin et urinaire sont les témoins de l'exposition récente aux dérivés solubles du nickel et de l'exposition récente et ancienne aux dérivés insolubles. Ces taux augmentent tout au long de la semaine de travail. Les valeurs d'exposition pour le nickel, concernant la population générale et les indices biologiques des personnes exposées en France, Allemagne et États-Unis, sont rapportées dans le Tableau II.

Le dosage du nickel dans les phanères a été proposé : il serait corrélé à l'intensité de l'exposition mais le risque de contamination externe rend difficile l'interprétation des résultats.

Le dosage du nickel dans la muqueuse nasale a été proposé, comme indicateur d'exposition.

Afin d'interpréter les résultats de la surveillance biologique, il est nécessaire d'identifier s'il s'agit d'une exposition aux dérivés solubles ou faiblement solubles du nickel (solubles : chlorure, sulfate et nitrate de nickel). L'excrétion urinaire étant rapide ( 1 à 2 jours après l'exposition), le nickel n'est pas un toxique cumulatif ; aussi les taux de nickel sérique ou de nickel urinaire sont considérés comme des indicateurs valables d'une exposition récente aux dérivés solubles du nickel. 
Tableau VI : Nickel (nickel métal, oxyde de nickel, carbonate et sulfure de nickel, minerais à base de sulfure) (30).

\begin{tabular}{|c|c|}
\hline $\begin{array}{c}\text { Concentration de Nickel } \\
\text { dans l'air } \\
\left(\mathrm{mg} / \mathrm{m}^{3}\right)\end{array}$ & $\begin{array}{c}\text { Nickel dans l'urine } \\
(\mu \mathrm{g} / \mathrm{l}) \\
\text { Moment de prélèv. : c }\end{array}$ \\
\hline 0,10 & 15 \\
\hline 0,30 & 30 \\
\hline $\mathbf{0 , 5 0}$ & $\mathbf{4 5}$ \\
\hline
\end{tabular}

\section{BERYLLIUM}

\section{Sources d'exposition}

Le béryllium est très utilisé sous toutes ses formes (métal : $30 \%$; alliages : $60 \%$; oxyde de béryllium : $5 \%)$

Les principales sources d'activités industrielles consommatrices de béryllium sont les industries électriques, les industries de l'électronique, l'aéronautique, la céramique, la métallurgie, la récupération des métaux précieux et les industries de la chimie, en prothèse dentaire.

\section{Métabolisme}

Le béryllium, ses oxydes et ses sels, toxiques surtout sous forme de poussières ou de vapeurs, pénètrent dans l'organisme essentiellement par voie pulmonaire, à un moindre degré par voie digestive $(<10 \%)$ et très faiblement par voie cutanée. L'importance de l'absorption dépend de la solubilité, de la dose et de la taille des composés minéraux. Le passage dans la circulation sanguine et faible. Le béryllium s'accumule dans le foie en cas d'exposition importante et à long terme dans le squelette et dans les ganglions lymphatiques. Environ $90 \%$ de la dose absorbée sont éliminés dans les urines jusque 10 jours après arrêt de l'exposition. La demi-vie du béryllium est inversement proportionnelle à la dose absorbée.

\section{Mécanisme de toxicité}

De nombreuses données laissent supposer que le béryllium se lie à une protéine jouant un rôle d'haptène. Ces complexes sont mis en évidence par une forte positivi- té du test de transformation lymphoblastique et à un degré moindre de l'inhibition de la migration des macrophages. La pathologie chronique semble reposer sur un mécanisme d'hypersensibilité à médiation cellulaire de type IV (27).

\section{Manifestations cliniques}

La bérylliose chronique peut être observée avec tous les composés du béryllium, mais l'oxyde de béryllium $(\mathrm{BeO})$ moins soluble que les sels est plus fréquemment en cause. C'est une maladie systémique (asthénie, amaigrissement, fièvre, arthralgies, sueurs, dyspnées, râles, toux....) $(28,29)$. Elle est dominée par une atteinte pulmonaire et peut s'accompagner de lésions cutanées, rénales (lithiases), osseuses (ostéomalacies). Le béryllium et ses composés sont classés parmi les agents cancérigènes pour l'homme.

\section{Surveillance biologique}

Les concentrations urinaires de béryllium reflètent le pool de béryllium mobilisé mais pas l'exposition récente ni la quantité de produit contenu dans l'organisme. Aucune donnée ne permet de corréler valablement les taux sanguins et urinaires à l'intensité de l'exposition. Le tabac augmente les concentrations.

\section{Conclusion}

La surveillance biologique de l'exposition constitue un progrès dans la démarche préventive dans des situations d'exposition professionnelle à des substances chimiques, ici les métaux. Complémentaire de la surveillance de l'environnement, elle permet d'affirmer l'évaluation de l'exposition des salariés au risque chimique lors du travail, en prenant en compte TOUTES les voies d'absorption.

La surveillance biologique peut :

- déterminer s'il y a risque d'absorption par voie cutanée et/ou par voie digestive

- venir à l'appui des contrôles d'exposition dans l'air des milieux du travail

- permettre d'évaluer l'efficacité d'équipement de protection collective ou industrielle

- déceler une exposition autre que professionnelle. 


\section{Références}

1. Lauwerys R.R. Toxicologie industrielle et intoxications professionnelles. 3ème édition. Paris : Masson, 1992.

2. Brondeau .T., Schneider O. Indicateurs biologiques d'exposition. Principes de base et valeurs-guides utilisables en France. Cahiers de notes documentaires, 1997, $\mathrm{n}^{\circ} 169$ : 589-595.

3. Conférence européenne de toxicologie environnementale à Munich, 16-18 juin 1993. Marqueurs biologiques d'exposition aux substances chimiques. Documents pour le médecin du travail, 1993, n55:283-286.

4. Ferrier V. Surveillance biologique : une nouvelle frontière. Travail et Sécurité, 1997, nº11 : 13-18.

5. La Surveillance biologique des expositions aux substances chimiques. Nouvel outil d'évaluation de l'exposition toxique professionnelle. Dr François Muller (AIMT 67). Mise à jour août 1999.

6. Pierre F. Surveillance biologique, de la théorie à la pratique. Cah. Notes Doc, 1989, 136 : 469-475.

7. Goodarzi F., Huggins FE. Monitoring the species of arsenic, chromium and nickel in milled coal, bottom ash and fly ash from a pulverized coal-fired power plant in western Canada. J. Environ. Monit., 2001, 3 (1) : 1-6.

8. Chappuis P. Techniques d'analyse des oligoéléments chez l'homme. Editions Médicales Internationales Lavoisier, 1995, Paris.

9. Expertise Collective INSERM. Le Plomb dans l'environnement. Quels risques pour la santé ? Les Editions INSERM 1999.

10. Wan B.J., Zhang Y., Tian C.Y., Cai Y., Jiang H.B. Blood lead dynamics of lead-exposed pregnant women and its effects on fetus development. Biomed. Environ. Sci., 1996, $9:$ :1-45.

11. Guidance of laboratory techniques in occupational medicine. Health ad safety executive. Occupational medicine and hygiene laboratory, 6th ed., Crown 1993.

12. Lauwerys R.R., Hoet P. Industrial Chemical Exposure. Guidelines for Biological Monitoring. Lewis publischers, 2nd ed, CRC Press, Inc 1993.

13. Duc Kaminsky M., Klein P. Intoxication par le plomb et ses sels. Encycl. Med. Chir. (Elsevier, Paris). Toxicologie - Pathologie professionnelle, 16-007-A-10, 1994 : 10.

14. BIOTOX. Guide biotoxicologique pour les médecins du travail. Inventaire des laboratoires effectuant des dosages biologiques de toxiques industriels. Edition INRS, décembre 1997, ED $791: 160$ p.

15. Bernard A., Lauwerys R. Cadmium. Encycl. Med. Chir. (Elsevier, Paris). Toxicologie - Pathologie professionnelle, 16-002-B-30, $1992: 4$

16. Javelaud B. Arsenic et dérivés. Toxicologie. . Encycl. Med. Chir. (Elsevier, Paris). Toxicologie - Pathologie professionnelle, 16-002-A-30, 1994.
17. Mandal BK, Ogra Y., Suzuki KT. Identification of Dimethylarsinous and Monomethylarsonous Acids in human urine of the arsenic-affected area in West Bengal, India. Chem Res. Toxicol., 200114 (4) : 371-378.

18. Bissen M., Frimmel FH. Speciation of As (III), As (V), MMA and SMA in contaminated soil extracts by HPLCICP/MS. Fresenius J. Anal Chem., 2000, 367 (1) : 5155.

19. Roussel C., Neel C., Bril H. Minerals controlling arsenic and lead solubility in an abandoned gold mine tailings. Sci. Total Environ., 2000, 263 (1-3) : 209-219.

20. Hoet P. Lauwerys R. Mercure et composés inorganiques. Encycl. Med. Chir. (Elsevier, Paris). Toxicologie Pathologie professionnelle, $1996: 7$.

21. Lohman K., Pai P., Seigneur C., Levin L. Sensitivity analysis of mercury human exposure. Sci Total Environ., 2000, $259: 3-11$.

22. WHO, World Health Organization. Chromium.World Organization, Environmental Health criteria 61, Geneva, $1988: 1-197$.

23. Ducros V. Le chrome. In : Chappuis Ph. - les oligoéléments en médecine et en biologie. Paris : Lavoisier, Tec et Doc, 1991, pp 499-522.

24. Meyer-Bisch C., Pham Q.T., Mur J.M., Massin N., Moulin J.J., Teculescu D., Carton B., Pierre F., Baruthio F., Respiratory hazards in hard metal workers : a cross sectional study. Brit. J. Ind. Med. 1989, 46, 302-309.

25. Sprince N.L., Oliver L. C., Eisen E.A., Greene R.E., Chamberlin R.I. Cobalt exposure and lung disease in tungsten carbide production. A cross-sectional study of current workers. Am. Rev. Respir. Dis., 1988, 138 : 12201226.

26. Sunderman F.W. Biological monitoring of nickel in humans. Cand J. Work Environ. Health. 1993, 19/ supplt $1: 34-38$.

27. Saltini C.,Winestock K., Kirby M., Pinkston P., Crystal R.G. Maintenance of alveolitis in patients with chronic beryllium disease by beryllium-specific helper $\mathrm{T}$ cells. $\mathrm{N}$. Eng. J. Med. , 1989, 320: 1103-1109.

28. Kriebel D., Brain J.D., Sprince N.L.,Kazemi H. The pulmonary toxicity of beryllium, state of the art. Am. Rev. Respir. Dis. 1988, 137 : 464-473.

29. Parkes W. R. Beryllium disease. In : Occupational lung disorders. Butterworths, Inc. London, Boston. 1982, pp 333-358.

30. Schneider O. et Brondeau M.T. Indices biologiques d'exposition. Cah. Notes Doc-Hygiène et sécurité au travail, $1999, \mathrm{n}^{\circ} 174$. 